\title{
Factores asociados a síndrome de boca dolorosa en una población de mujeres mexicanas y su relación con el climaterio
}

\author{
Castillo $\mathrm{F}^{*}$, Aldape $\mathrm{B}^{* *}$
}

\section{RESUMEN}

Objetivo: Identificar los factores asociados a SBD presentes en una población de mujeres mexicanas, para poder así obtener un diagnóstico acertado con el que sea posible brindar un mejor tratamiento a este padecimiento.

Métodos: Se aplicó una historia clínica especial para pacientes con SBD, en aquellos pacientes en quienes se sospecha del padecimiento. Además se llevó a cabo la exploración bucal en busca de datos clínicos y estudios de laboratorio, incluyendo niveles estrogénicos en sangre. Se estudiaron e identificaron los factores locales, sistémicos y psicogénicos en cada paciente. Se hicieron las remisiones correspondientes y se aplicó un tratamiento alternativo con infusión de nopal y capsaicina.

Resultados: De un total de 833 pacientes que se revisaron de enero del 2001 a diciembre del 2002, fueron diagnosticados 13 sujetos con SBD. De éstos, 11 fueron mujeres de 5 a 83 años de edad. El 90,9\% de las pacientes presenta depresión y ansiedad, además de otros trastornos emocionales como problemas intrafamiliares. En el $81,8 \%$ se observó hiposalivación y presencia de polimedicación. Se obtuvo también la cuantificación de estrógenos en sangre en siete pacientes, de las cuales seis $(85,7 \%)$ presentan déficit estrogénico (10$50 \mathrm{pg} / \mathrm{ml})$.

Conclusiones: El factor psicogénico fue el más frecuente en esta población, seguido por hiposalivación y factores locales irritantes. Esto es debido, probablemente, a aspectos socio-económicos y culturales propios de la mujer mexicana, los cuales se suman a las alteraciones posmenopáusicas por déficit estrogénico y factores propios de la edad, provocando desorden emocional y falta de atención médica y buco-dental.

Palabras clave: Síndrome de boca dolorosa, Climaterio, Hiposalivación, Medicamentos.

\section{SUMMARY}

Objective: To identify the associated factors to present BMS in a population of Mexican women, to thus be able to obtain a diagnosis right with that it is possible to offer a better treatment to this.

Methods: A clinical history for patients was applied special for BMS, those patients in those who she suspects herself the BMS. In addition it was carried out the oral exploration in search of clinical data and studies of laboratory, including estrogenic levels in blood. They studied e they identified the local, systemic and psychogenic factors in each patient. The corresponding remissions became and a treatment was applied alternative with infusion of nopal and capsaicina.

Results: Of a total of 833 patients, 13 subjects with BMS were diagnosed. Of these 11 women, of 51 to 83 years of age. The $90.9 \%$ of the patients present/display depression and anxiety, in addition to other emotional upheavals like problems intrarelatives.

Conclusions: The psychogenic factor was most frequent in this population, followed by irritating hypo salivation and local factors. This is probably had own to socioeconomic and cultural aspects of Mexican woman, which are added to the postmenopausal alterations by deficit estrogenic and own factors of the age, causing emotional disorder and lack of medical and dental attention. 
Key words: Burning Mouth Syndrome, Climacteric, Hiposalivation, Medicaments.

Aceptado para publicación: Octubre 2005.

* Cirujano Dentista

** Profesora de Patología Bucal.

Facultad de Odontología, UNAM

Castillo F, Aldape B. Factores asociados a síndrome de boca dolorosa en una población de mujeres mexicanas y su relación con el climaterio. Av. Odontoestomatol 2006; 22 (3): 177-185.

\section{INTRODUCCION}

Esta es una entidad patológica caracterizada por una sensación de ardor bucal en una mucosa clínicamente sana, y cuya etiología permanece incierta. El propósito de este estudio fue identificar los factores etiológicos en un grupo de mujeres mexicanas diagnosticadas con SBD, se explicó el papel físico y psicológico que juega el climaterio en la sintomatología de ardor bucal, y también se elaboró una historia clínica especial para pacientes con este trastorno.

La prevalencia de SBD en la población general varía del 2,6\% a 3,7\%, ambos resultados de países desarrollados $(1,2)$. Al hablar de la población que visita un centro de salud bucal, el porcentaje se hace mayor; en Argentina se encontró una prevalencia del $13 \%$ (3). El SBD afecta más frecuentemente a mujeres que a hombres, con una razón de 3-4:1 $(1,4)$. Lamey PJ y Lewis MAO reportan una razón de $7: 1$, sin embargo no excluyen lesiones bucales (5). La prevalencia en mujeres varía del 4,2\% al 5,5\% mientras que en hombres es del $0,8 \%$ al $1,6 \%$ (6). La edad promedio de aparición es a los 60 años (4).

La sensación quemante y dolorosa del SBD se cuantifica comúnmente por medio de escalas como la "Visual Analogue Scale" (VAS), en la que se pide al paciente calificar la intensidad del dolor bucal en un rango del 0 al 10, donde 0 es ausencia de dolor y 10 es un dolor extremo. En pacientes suecos se ha usado la escala visual análoga con un resultado de 3.8, en franceses con 6,2 y en italianos con 5,1 $(2,6)$. El dolor puede durar de meses a años con un promedio de 4,6 a 6,4 años (7).

El ardor puede ocurrir en muchos sitios dentro de la cavidad bucal, ya sea con carácter localizado o generalizado. El sitio más comúnmente afectado es la lengua, principalmente la punta y los dos tercios anteriores con variaciones de un $50 \%$ a $78 \%$. Los lugares que le siguen en orden descendente son el paladar duro, labios, procesos alveolares y mucosa bucal. Con menor frecuencia se encuentra el piso de boca, paladar blando y garganta. La distribución de la sintomatología puede dar una pista de los posibles factores etiológicos locales $(1,4,5,8)$.

Existen tres tipos de SBD, el SBD tipo I y II son los más frecuentes, en los que el dolor esta presente todos los días, mientras que en tipo III, el dolor tiene un carácter intermitente. En el SBD tipo I el paciente no sufre ninguna sintomatología al despertarse en las mañanas, pero la sensación ardorosa comienza progresivamente y se acentúa conforme pasa el día, hasta exacerbarse por la tarde. Cuando la sintomatología está presente al despertarse y permanece constante durante todo el día sin variaciones se habla de un SBD tipo II. En el tipo III existen días enteros en que desaparece el ardor, localizándose en lugares poco comunes, como piso de boca y garganta $(4,5)$. 
Factores etiológicos de SBD (1,5,7-14):

\section{- Factores locales}

- Irritación mecánica.

- Condición dental y protésica.

- Hábitos parafuncionales.

- Hiposalivación.

- Alergia a materiales dentales.

- Lengua geográfica.

- Reflujo esofágico.

\section{- Factores Sistémicos}

- Desorden nutricional.

- Anemias.

- Deficiencias vitamínicas.

- Medicación y polimedicación.

- Diabetes mellitus.

- Síndrome de Sjögren.

- $\mathrm{VIH} / \mathrm{SIDA}$.

\section{- Factores Psicogénicos}

- Depresión.

- Ansiedad.

- Estrés.

- Alteraciones del sueño.

- Problemas intrafamiliares, laborales y económicos.

- Historia de evento traumático.

En mujeres posmenopáusicas, la prevalencia de ardor bucal ha llegado a ser hasta de un $93 \%$ según Massler $M$, sin embargo, en estudios mejor controlados y más recientes se reportan prevalencias más acertadas como la de Basker RM del 26\%, Ferguson MM del 17,9\% y Wardrop RW del 33\%. Estos porcentajes son contrastantes y mucho más altos que la prevalencia de ardor bucal en la población general que es del $5,1 \%$, o en mujeres premenopáusicas del $6 \%(1,12,13,15)$.

El climaterio es la fase del envejecimiento de la mujer que señala la transición entre la fase reproductiva y la no reproductiva de la vida, entre los 35 y 65 años de edad, determinada por la declinación de la función ovárica. La menopausia es el periodo menstrual final y ocurre durante el climaterio, al agotarse los folículos primarios; sólo se considera como tal des- pués de que haya pasado un año o más sin nuevos sangrados. En EU y Europa se presenta hacia los 50 años de edad y en México hacia los 47, en promedio, aunque puede suceder entre los 42 y 52 años. La menopausia artificial es el cese de los periodos menstruales mediante cirugía o radiación, la cual produce la misma sintomatología que la menopausia natural, solamente que en forma brusca e intensa cuando se realiza en pacientes jóvenes con función ovárica adecuada $(16,17)$.

La premenopausia puede comenzar después de los 35 años de edad, en que el ovario disminuye de tamaño y contiene menos ovocitos y estructuras foliculares y más folículos atrésicos en degeneración. Esta pérdida de ovocitos y folículos provoca una disminución gradual de los estrógenos, la cual, alrededor de cinco años antes de la menopausia, trae como consecuencia la elevación de la hormona foliculoestimulante (FSH), que es la primera señal de laboratorio de la premenopausia. Posteriormente se producen ciclos anovulatorios, con el consiguiente cese de formación de cuerpos lúteos y, al no haber producción de progesterona, hay sangrados genitales anormales en su cantidad, frecuencia y duración, siendo éstos los primeros datos clínicos de la premenopausia, junto con bochornos y sudoración profusa. Los estrógenos en cantidades muy bajas son incapaces de estimular el crecimiento endometrial, el cual se atrofia con el tiempo y suspende el sangrado. Esta etapa del climaterio comienza después de ocurrir la menopausia y su duración es variable, pudiendo ser tan corta como 5 años o tan larga como 15 años (16-18).

Tan pronto como empieza la posmenopausia y como consecuencia del impacto psicológico que ocasiona la supresión definitiva del sangrado genital, la mujer posmenopáusica puede verse afectada de forma leva a severa, por insomnio y en ocasiones somnolencia, estados de depresión y ansiedad, fatiga, mareos, irritabilidad, sentimiento de insuficiencia y falta de realización, temor al envejecimiento y a la muerte, temor a perder la satisfacción sexual o a que el esposo pierda el interés por ella, cefalea, disnea y fluctuaciones en el estado de ánimo $(16,17)$. En México y países occidentales, los trastornos emocionales son consecuencia de la sintomatología general y del entorno personal, familiar, laboral y social en el que se 
desenvuelve la mujer. Se ha reportado que en mujeres europeas y orientales las repercusiones psicológicas son menores (19).

Los signos y síntomas se atribuyen al medio estrogénico disminuido (10-50 pg/ml de estradiol en plasma, siendo que en edad reproductiva puede ser > 200 pg/ml), provocando alteraciones a nivel del aparato genitourinario, mamas, piel y mucosas, sistema cardiovascular y sistema óseo $(16,17)$. Las alteraciones en cavidad bucal son el aumento en la incidencia de enfermedades periodontales, xerostomía, enfermedades como liquen plano, síndrome de Sjögren y SBD. Grushka $M$ menciona que en mujeres posmenopáusicas con SBD puede encontrarse ardor bucal en el $43 \%$, alteración del gusto en el $27 \%$, o ambos síntomas en el $30 \%$ de las pacientes. Además, la piel y mucosas se ven afectadas por sequedad, prurito, son fácilmente traumatizadas, pierden elasticidad y flexibilidad $(4,18)$.

La terapia hormonal de reemplazo (THR) proporciona beneficios en cuanto al alivio de los signos y síntomas climatéricos como bochornos, insomnio, dispareunia por atrofia vaginal senil y osteoporosis, proporcionando a la paciente una mejor calidad de vida. También protege contra la arterioesclerosis cardiovascular, reduce los infartos del miocardio y la coronariopatía mortal (16). En México, el uso de estrógenos es más limitado debido a la baja frecuencia de diagnóstico de problemas asociados al climaterio, insuficiente promoción educativa para la población general y a que la mujer mexicana está expuesta a información de medios de divulgación popular. Lo anterior impacta desfavorablemente en una menor aceptación y continuidad de uso necesaria para obtener beneficios potenciales en salud y calidad de vida para la mujer (19). El papel de la THR en la sintomatología bucal no se ha clarificado hasta nuestros días. Parece mejorar la cantidad y calidad de la función de las glándulas salivales en mujeres premenopáusicas y posmenopáusicas. Forabosco A encuentra que las mujeres con sintomatología ardorosa y receptores de estrógenos en mucosa bucal responden al reemplazo hormonal, cosa que no sucede en las pacientes que carecen de estos receptores, sin embargo, no es posible asegurar que la THR sea un tratamiento efectivo para la sintomatología bucal (20).
Tratamientos alternativos para SBD:

- Capsaicina (terapia desensibilizante).

- Infusión de nopal (sustituto de saliva).

- Saliva artificial.

- Guarda oclusal.

- Suplemento vitamínico.

\section{METODOLOGÍA}

En el estudio se incluyeron pacientes de género femenino que presenten la sintomatología característica del síndrome de boca dolorosa y que a la inspección clínica presenten mucosa clínicamente normal con ausencia de signos clínicos de lesión. Se excluyeron pacientes de género masculino, hayan sido o no diagnosticados con síndrome de boca dolorosa, y pacientes de género femenino quienes refieren tener ardor en alguna parte de la cavidad bucal, pero en quienes se hayan encontrado signos clínicos de lesión durante la exploración.

Los factores que se investigaron en cada paciente son: edad, estado civil, nivel del dolor (escala análoga visual), circunstancias bajo las cuales comienza la sintomatología, etapa del climaterio en que se encuentra la paciente, tipo de menopausia, hiposalivación, bordes cortantes y restauraciones desajustadas, hábitos parafuncionales, polimedicación, fármacos predisponentes de hiposalivación y ardor bucal, eritropenia, hiperglucemia, déficit estrogénico, depresión y ansiedad, problemas intrafamiliares, insomnio y cancerofobia.

La obtención de datos en este estudio se hizo por medio de la Historia Clínica elaborada especialmente para pacientes que padecen SBD. En ella se incluyen un detallado interrogatorio, exploración bucal y estudios complementarios principalmente. Dentro del interrogatorio directo que se le hace a la paciente se indaga sobre datos demográficos, características de la sintomatología bucal, nivel del dolor (escala visual análoga de 0 a 10), antecedentes dentales, heredo-familiares, personales patológicos y no patológicos, así como también antecedentes quirúrgicos, farmacológicos, ginecológicos y psicológicos. El interrogatorio consta de preguntas específicas encaminadas al cumplimiento de los objetivos, ya que, 
de no contar con un formato ya establecido, sería muy difícil la identificación de los factores asociados a SBD.

Al terminar el interrogatorio, se procede a hacer la exploración bucal registrando el estado de los tejidos blandos, órganos dentarios, materiales de restauración y ATM. Se cuantifica la salivación por medio de la sialometría. Se solicitan los estudios de gabinete pertinentes, de los cuales son básicos una ortopantomografía, biometría hemática, química sanguínea y nivel estrogénico en sangre.

Una vez obtenidos todos los datos necesarios es posible emitir el diagnóstico indicando el tipo de SBD que presenta la paciente, así como los factores locales, sistémicos y psicogénicos identificados. Según el caso se hace la remisión o interconsultas necesarias, ya sea a nivel odontológico, médico o psicológico, con el fin de que la paciente reciba un tratamiento integral que disminuya su exposición a los factores etiológicos.

\section{RESULTADOS}

De los 833 pacientes, 13 sujetos fueron diagnosticados con SBD, de los cuales 11 son pacientes de género femenino y 2 del masculino. La prevalencia de SBD en este estudio fue del 1,56\% (Gráfico 1).

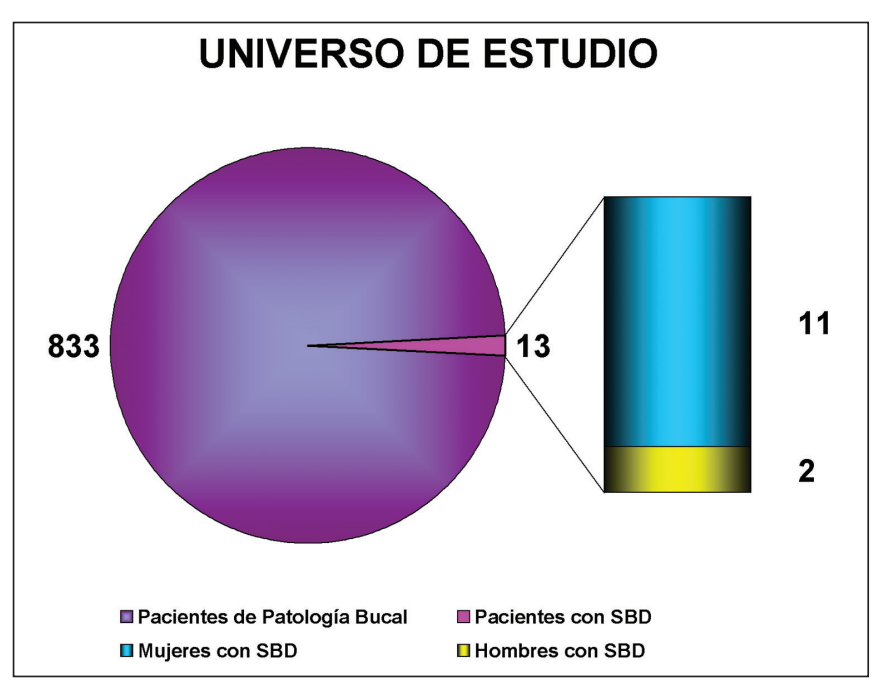

Gráfico 1.
Los tipos de SBD que se encontraron en este estudio fueron dos: el tipo I que se presentó en 5 de 11 pacientes $(45,5 \%)$ y el SBD tipo II que se presentó en 6 de 11 pacientes (54,5\%). No hubo ningún caso de SBD tipo III. Es decir, hay más pacientes que sufren los síntomas desde que se despiertan hasta que se van a dormir.

La edad promedio de las once pacientes fue de 65 años. Se observó que la séptima década de la vida fue la más afectada, y la menos fue la novena. Predominaron las pacientes viudas $(45,5 \%)$, sobre las casadas $(36,4 \%)$ y las divorciadas $(18,2 \%)$. Las pacientes de este estudio fueron mujeres en la etapa de la posmenopausia $(54,5 \%)$ y vejez $(45,5 \%)$. Al comparar los tiempos de evolución del dolor en cavidad bucal y el tiempo transcurrido desde que ocurrió la menopausia, se observa que el 99,9\% de las pacientes tienen más tiempo de haber experimentado la menopausia que el dolor, y sólo una reporta que la menopausia y la sintomatología bucal comenzaron a la par. Predominan las pacientes cuya evolución del dolor no supera los 12 meses (54,5\%), sin embargo, hubo quien ha soportado la sintomatología por 10, 15 y hasta 20 años. Un dato interesante es que cuatro de las pacientes $(36,4 \%)$ tuvieron su menopausia de manera artificial o quirúrgica, practicándoseles la histerectomía entre los 40 y los 44 años de edad. A este grupo pertenece la paciente cuyos tiempos de evolución de la menopausia y del dolor coinciden (Tabla 1).

A pesar de que la sensación ardorosa puede involucrar cualquier sitio de la cavidad bucal, la lengua es por mucho el sitio de localización más frecuente, ya sea sola $(45,5 \%)$ o con múltiples áreas involucradas (54,5\%). El 81,8\% refiere que ésta es la localización más afectada. En cuanto a las circunstancias bajo las cuales comenzó el dolor, predominando las mujeres que relacionan el comienzo de su padecimiento a un suceso de orden emocional, como son la muerte del cónyuge y problemas intrafamiliares crónicos (54,5\%). En una de ellas fue a raíz de un tratamiento dental, específicamente un curetaje periodontal, según refiere la paciente. La tabla 2 describe los diferentes factores asociados a SBD que se encontraron en las pacientes. El factor local más frecuente fue la disminución de la secreción salival en el 81,8\% de los casos. Los factores sistémicos fueron difíciles 


\section{TABLA 1.- DOLOR BUCAL Y MENOPAUSIA}

\begin{tabular}{|c|c|c|c|c|c|}
\hline \multicolumn{2}{|c|}{$\begin{array}{l}\text { Dolor } \\
\text { (años) }\end{array}$} & \multicolumn{2}{|c|}{$\begin{array}{l}\text { Menopausia } \\
\text { (años) }\end{array}$} & \multicolumn{2}{|c|}{$\begin{array}{l}\text { Tipo de menopausia } \\
\text { (Núm. de casos) }\end{array}$} \\
\hline $\begin{array}{c}\text { Edad } \\
\text { promedio } \\
\text { en que se } \\
\text { presentó }\end{array}$ & $\begin{array}{c}\text { Rango } \\
\text { de duración }\end{array}$ & $\begin{array}{c}\text { Edad } \\
\text { promedio } \\
\text { en que se } \\
\text { presentó }\end{array}$ & $\begin{array}{l}\text { Rango } \\
\text { desde que } \\
\text { ocurrió }\end{array}$ & Natural & Artificial \\
\hline 61 & $0,5-20$ & 45,5 & $5-40$ & 7 & 4 \\
\hline
\end{tabular}

de determinar debido a que el $36,4 \%$ de las pacientes no obtuvo sus estudios de laboratorio, sin embargo, es posible indicar que seis de siete pacientes $(85,7 \%)$ presentan déficit estrogénico con niveles de estrógenos en sangre de 10 a 50 pg/ml. Se encontró que el $81,8 \%$ están polimedicadas ( 2 a 7 fármacos) y en promedio, cada paciente toma 2,63 fármacos. Además, nueve pacientes $(81,8 \%)$ toman algún fármaco predisponente de hiposalivación y son éstas quienes precisamente la padecen. Dentro de los trastornos psicogénicos, la depresión y la ansiedad fueron los más comúnmente encontrados (99,9\%).

De los fármacos que utilizaban las pacientes, el $80 \%$ provoca reacciones adversas en cavidad bucal, ya sea hiposalivación, ardor o alteración del gusto (disgeusia). El medicamento que más comúnmente tomaban las pacientes fue clonazepam (Rivotril) del grupo de los antiepilépticos, prescrito para el tratamiento de la ansiedad y depresión según refieren. Las pacientes que utilizan antihipertensivos toman captopril (Capotena), inhibidor de la ECA cuyo principal efecto adverso en cavidad bucal es el ardor. Una de las pacientes bajo terapia hormonal tomaba tibolona (Livial), esteroide sintético para el tratamiento de los síntomas posmenopáusicos. Ella refiere haber desaparecido el dolor bucal al suspender el uso de éste.

En este estudio se evaluó el nivel del dolor con la escala visual análoga (VAS). Se observó que el nivel del dolor al momento de realizar la historia clínica fue mayor de 8 en ocho de las pacientes (72,7\%), tres de las cuales refieren padecer un nivel de ardor máximo de 19, El nivel es de 4 a 7 en tres pacientes $(27,3 \%)$. Ninguna paciente refiere un nivel del dolor menor de 3. Como hallazgo complementario, mas no como resultado del estudio, se muestra el nivel del dolor que presentaron las pacientes para la última consulta después del tratamiento alternativo, a base de infusión de nopal y capsaicina. En nueve pacientes el nivel del dolor bajó considerablemente,

\section{TABLA 2.- FACTORES LOCALES, SISTÉMICOS Y PSICOGÉNICOS}

\begin{tabular}{l|c|c|}
\hline & $\begin{array}{c}\text { Núm. de casos/ } \\
\text { total pacientes }\end{array}$ & $\%$ \\
\hline $\begin{array}{l}\text { Factores locales } \\
\text { - Xerostomía }\end{array}$ & $9 / 11$ & 81,8 \\
- Bordes cortantes, & & \\
$\quad$ restauraciones, & & \\
prótesis & $8 / 11$ & 72,7 \\
- Hábitos parafuncionales & $7 / 11$ & 63,6 \\
Factores sistémicos & & \\
- Polimedicación & $9 / 11$ & 81,8 \\
- Fármacos predisponentes & & \\
de xerostomía & $9 / 11$ & 81,8 \\
- Fármacos predisponentes & & \\
de ardor bucal & $2 / 11$ & 18,2 \\
- Eritropenia & $2 / 7$ & 28,6 \\
- Hiperglucemia & $0 / 7$ & 0,0 \\
- Déficit estrogénico & $6 / 7$ & 85,7 \\
Factores psicogénicos & & \\
- Depresión y ansiedad & $10 / 11$ & 90,9 \\
- Problemas interfamiliares & $7 / 11$ & 63,6 \\
- Insomnio & $7 / 11$ & 63,6 \\
- Cancerofobia & $6 / 11$ & 54,5 \\
\hline
\end{tabular}




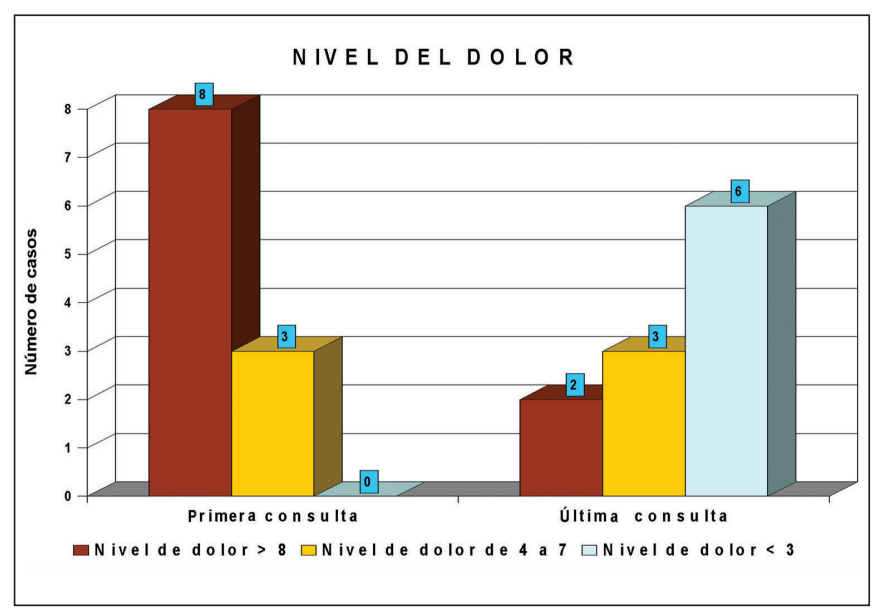

Gráfico 2.

siendo menor de 3 para seis de ellas (54,5\%). En dos pacientes permaneció en el mismo nivel de $8(18,2 \%)$, sin embargo ninguna tuvo nivel máximo de 10 y en ninguna aumentó el dolor.

\section{DISCUSIÓN}

Se encontró una prevalencia de $1,56 \%$ del total de pacientes revisados. En comparación con los resultados obtenidos por Hakeberg $M$ y Bergdahl $M$ del $3,9 \%$ y $3,7 \%$ respectivamente, se observa que la cifra en nuestro estudio es menor, a pesar de que no fue obtenida de la población en general, sino de pacientes que acuden a un centro de especialidades odontológicas. La relación mujeres a hombres es de 6,5:1, lo cual es contrastante con el 3:1 de Basker RM y Maresky LS y muy próximo al 7:1 de Lamey PJ. La edad promedio en que se presentó el SBD en las pacientes fue de 65 años, lo cual es ligeramente superior a la obtenida por autores como Maresky LS, Grushka My Bergdahl M, con un resultado de 62, 60 y 57 años respectivamente.

Hakeberg $M$ encuentra que el nivel del dolor en sus pacientes con SBD según la escala visual análoga es de 3,8; en el presente estudio se obtuvo un nivel del dolor muy superior, de 8.5 con la misma escala, lo cual lleva a pensar acerca de la gravedad de los casos de SBD que tenemos en México, aunado a que la prioridad de la mujer mexicana no es buscar atención médica, y mucho menos dental.
Los resultados de este estudio concuerdan con los obtenidos por Browning S, Lamey PJ, Ship J y Bergdahl $M$, quienes mencionan que los pacientes con SBD sufren de problemas psicogénicos. Sin embargo, la cifra de pacientes con alteraciones psicogénicas que se obtuvo fue del 99,9\%, muy superior al $44 \%$ que encuentra Browning $\mathrm{S}$. Esto se debe a que el estudio de este autor fue encaminado exclusivamente a la detección de desórdenes psiquiátricos, llevando a cabo evaluaciones psiquiátricas de orden familiar, personal, social, médico y mental por medio de un especialista en psiquiatría.

Al igual que Grushka M, Gorsky M, Maresky LS y Basker RM, nuestros resultados muestran que la hiposalivación es un factor que afecta frecuentemente a los pacientes que padecen SBD.

La medicación como factor etiológico sistémico es un tema variado y difícil. Autores como Hakeberg M encuentran que el consumo de medicamentos antihipertensivos, sedantes y hormonas sexuales es predisponente de sintomatología bucal. Gorsky M., muestra en sus resultados que el fármaco más consumido fue clordiazepoxide (Librium) del grupo de las benzodiazepinas. En cambio, Tarkkila L. y Bergdahl M., indican que los más utilizados fueron la terapia hormonal de reemplazo y las tiroxinas, respectivamente. En el presente estudio se encontró que el medicamento que más frecuentemente tomaron las pacientes fue clonazepam (Rivotril), antiepiléptico que provoca hiposalivación, entre otros efectos adversos.

Con respecto a las alteraciones menopáusicas como factores de riesgo del SBD, en este estudio se encontró, al igual que Massler $M$, Ferguson $M M$, Wardrop RW y Frutos R que durante el climaterio, específicamente en la posmenopausia, se ve incrementada la sintomatología bucal que experimentan las mujeres.

A pesar de que no haber sido parte del estudio, la terapéutica con sialogogos e infusión de nopal en nuestras pacientes mexicanas fue exitosa para combatir la hiposalivación en las ocho pacientes que siguieron las recomendaciones. En contraste, Gorsky M., administra $5 \mathrm{mg}$ cuatro veces al día de pilocarpina hidroclorada (Pilocar) para estimular la salivación, obteniendo resultados insatisfactorios. El tratamien- 
to alternativo a base de nopal ofrece buenos resultados y beneficios múltiples, además de su bajo costo y fácil adquisición en nuestro país, a diferencia de los sustitutos de saliva utilizados en otros países.

El presente estudio ofrece resultados descriptivos mas no significativos sobre los factores asociados a SBD. Sin embargo, a pesar de las limitaciones presentes, el estudio proporciona un panorama general sobre el SBD en una población de mujeres mexicanas, sus factores asociados, prevalencia, características clínicas, y terapéutica disponible.

\section{CONCLUSIONES}

- Este estudio sugiere que el grupo de factores etiológicos psicogénicos, es el más frecuentemente encontrado en las pacientes que padecen SBD, siendo la depresión y la ansiedad los desórdenes psicogénicos más comunes.

- Dentro de los factores etiológicos locales, la hiposalivación es el más frecuente, seguida por la presencia de bordes cortantes, restauraciones o prótesis desajustadas.

- La polimedicación y el uso de fármacos predisponentes de hiposalivación son los factores asociados más frecuentes dentro del grupo de factores etiológicos sistémicos.

- Se sugiere que la deficiencia estrogénica no es un factor determinante de SBD, sin embargo es el principal agente fisiopatológico responsable de los trastornos característicos del climaterio, y con ello, de la alteración psicogénica productora en gran parte, de la sintomatología de SBD.

- La sintomatología del SBD es el resultado de la interrelación de múltiples factores locales, sistémicos y psicogénicos, por lo que se requiere de la atención de varias especialidades médicas, y por lo tanto de recursos económicos considerables para mantener un tratamiento integral, situación que es difícil conseguir en México.

- El conocimiento de los factores de riesgo de SBD, así como el uso de una historia clínica específica para estos pacientes, son la base de una identificación precisa de factores etiológicos, y por lo tanto de un diagnóstico acertado necesario para dar un mejor tratamiento.

- Los problemas sociales, culturales y económicos que aquejan a las mujeres mexicanas difieren de aquellos que puedan padecer las mujeres de otros países, como las del primer mundo por ejemplo, donde el nivel de vida es más alto, la ideología no es tan conservadora, y las condiciones laborales y familiares no exigen tanto desgaste físico y emocional por parte de la mujer.

\section{BIBLIOGRAFÍA}

1. Basker RM, Sturdee DW, Davenport JC. Patients with burning mouth: a clinical investigation of causative factors, including climacteric and diabetes. Br Dent J 1978;145:9-16.

2. Bergdahl M, Bergdahl J. Burning mouth syndrome: prevalence and associated factors. J Oral Pathol Med 1999;28:350-4.

3. Ceccotti EL, Yasnig FH, Zanuso LH, Bernal MP. Abordaje interdisciplinario en pacientes con síndrome de ardor bucal. Bol AN de Medicina 1996; 74:441-7.

4. Grushka M. Clinical features of burning mouth syndrome. Oral Surg Oral Med Oral Pathol 1987; 63:30-6.

5. Lamey PJ, Lewis MAO. Oral medicine in practice: burning mouth syndrome. Br Dent J 1989; 167:197-200.

6. Hakeberg M, Berggren U, Hägglin C, Ahlqwist M. Reported burning mouth symptoms among middle-aged and elderly women. Eur J Oral Sci 1997;105:539-43.

7. Maresky LS, Bijl P, Gird I. Burning mouth syndrome: evaluation of multiple variables among 85 patients. Oral Surg Oral Med Oral Pathol 1993;75:303-7. 
8. Gorsky M, Silverman S, Chinn H. Clinical characteristics and management outcome in the burning mouth syndrome. Oral Surg Oral Med Oral Pathol 1991;72:192-5.

9. Cibirka R, Nelson S, Lefebvre C. Burning mouth syndrome: a review of etiologies. J Prosthet Dent 1997;78:93-7.

10. Ship J, Grushka M, Lipton J, Mott A, Sessle B, Dionne R. Burning mouth syndrome: an update. J Am Dent Assoc 1995;126(7):842-53.

11. Lamey PJ, Lamb AB. Prospective study of aetiological factors in burning mouth syndrome. Br Med J 1988;296:1243-6.

12. Ferguson MM, Carter J, Boyle P, Hart D, Lindsay $\mathrm{R}$. Oral complaints related to climacteric symptoms in oophorectomised women. J Roy Soc Med 1980; 74:492-8.

13. Massler M. Oral manifestations during the female climacteric: the postmenopausal syndrome. Oral Surg Oral Med Oral Pathol 1951;4: 1234-43.

14. Browning S, Scully C, Shirlaw P. The association between burning mouth syndrome and psychosocial disorders. Oral Surg Oral Med Oral Pathol 1987;64:171-4.

15. Wardrop RW, Hailes J, Burger H, Reade PC. Oral discomfort at menopause. Oral Surg Oral Med Oral Pathol 1989;67:535-40.
16. Jones H, Colston A, Burnett L. Tratado de ginecología de Novak. $11^{\mathrm{a}}$ ed. Interamericana; 1991. p. 349-86.

17. Instituto Mexicano del Seguro Social. Ginecología y obstetricia. $3^{a}$ ed. Méndez editores; 1998. p.739-45, 1009-29.

18. Frutos R, Rodríguez S, Miralles L, Machuca G. Manifestaciones orales y manejo odontológico durante la menopausia. Medicina Oral 2002; 7:26-35.

19. Velasco V, Pozos JL, Cardona JA. Terapia hormonal de reemplazo en el climaterio y la posmenopausia. Rev Med IMSS 2000; 38(2):131-38.

20. Forabosco A, Criscuolo M, Coukos G, Uceli E, Weinstein R, Botticelli A y col. Efficacy of hormonal replacement therapy in postmenopausal women with oral discomfort. Oral Surg Oral Med Oral Pathol 1992;73:570-4.

\section{CORRESPONDENCIA}

C.D. FátimaY. Castillo

Paralela, 2-13

Col. José MA. Pino Suárez

MEXICO 01140 D.F.

55162268

castillo_fat@yahoo.com 